\title{
The application of vorticity concepts to three irrigation intake designs
}

\author{
K. J. W. SPURR \& F. J. BOUCHERAT
}

\section{Mr H. R. Lupton, Consulting Engineer}

I sympathize with the Authors' aim to probe the process of the evening out of the distribution of the kinetic energy of the water so as to render its flow more palatable to the pumps, in particular by analysis of the birth, extent and extinction of the vortices which arise. It seems to me that for the practical analysis of vortex wanderings and mutual reactions to be effective more precise information is necessary

(a) on the mini-flow propeller probes and their orientation, so that it may be clear exactly what velocities are being measured

(b) on the exact positions of the propeller probes relative to the surfaces and edges which affect the vortices

(c) on the effect of small changes in the position and the orientation of the probes, especially where the region concerned may, recurrently, find itself in close proximity to the surface traced by a travelling vortex line or series of vortex lines; in such a region the velocities measured, and their gradients, are affected by, and may be indicative of, the nature of the micro-vortices, but for information so derived to be meaningful vortex formation conditions must be reasonably repetitive, which seems unlikely in the case considered by the Authors.

Even if this information were obtained, I think it would be very difficult to translate the vortical behaviour of the model to that of the full-size installation. I am therefore doubtful of the effective relevance of the spectral analysis of the flow at certain selected points and duties.

46. It is also necessary to know the design and size of the propeller meters in order to be able to estimate their sensitivity to components of the flow in directions other than the streamwise direction. Could the Authors give details of the actual readings obtained, and not merely the coefficients derived from those readings, to make clear the method of analysis applied?

47. Am I correct in understanding that the sole object of the spectral analysis is to demonstrate that during flow along a channel, and in particular past vertical columns and over floor sills, large vortices are broken up and succeeded by a larger number of smaller vortices? This would seem to be obvious without the aid of immersed instrumentation which must itself affect the flow regime under investigation.

48. Has any research been carried out to assess the value of the vortex-modifying devices in effecting an improvement in the feed flow to the pumps, i.e. has there been any measurement of pumping efficiency with and without the devices? I am inclined to

Paper published: Proc. Instn Civ. Engrs, Part 2, 1976, 61, June, 311-330. 

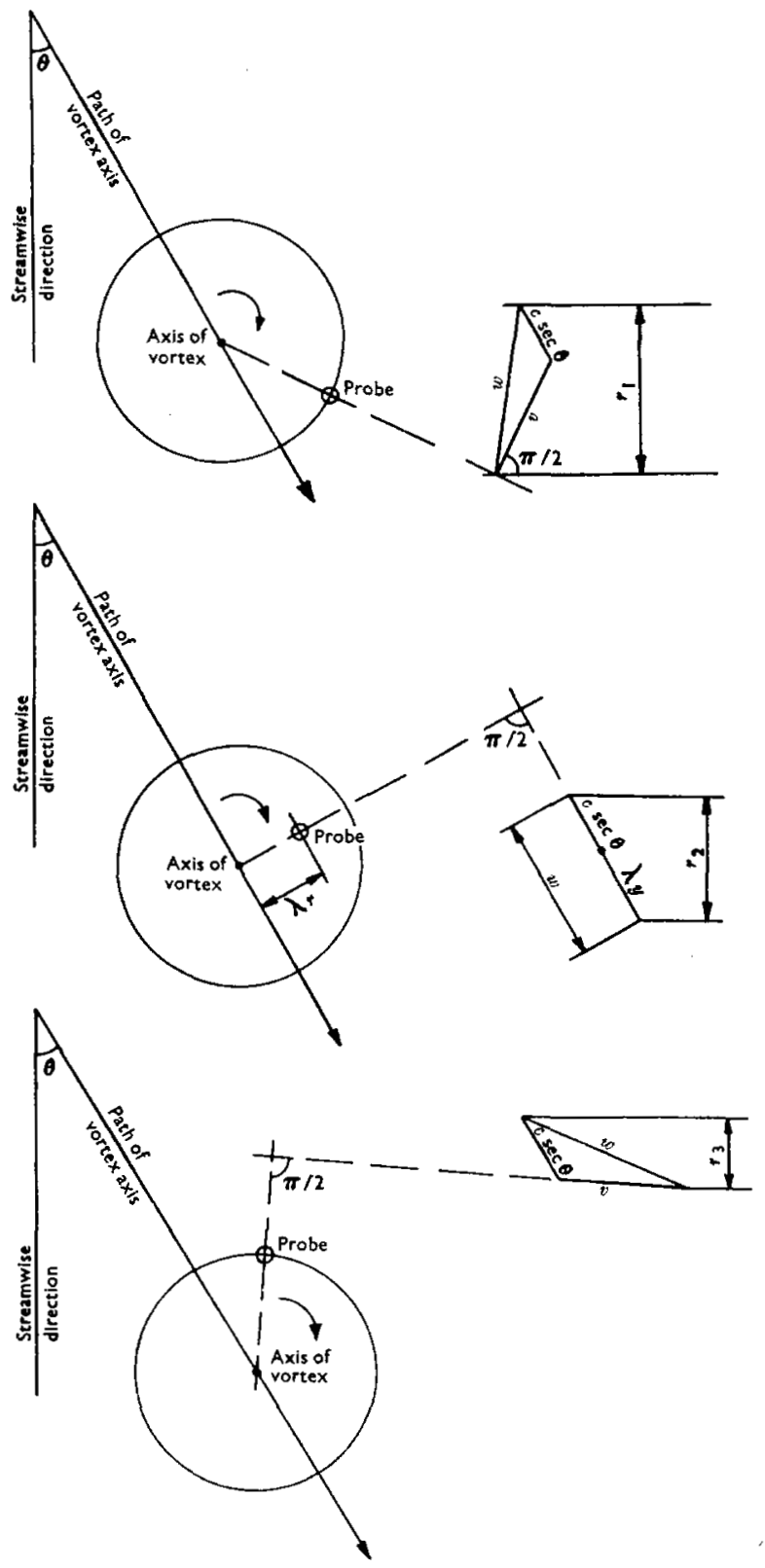

Fig. 19 
think that the coarse and fine screens are far more effective than the vertical columns and floor sills. Where screens are absent or a long way from the pumps, the devices described may be important. Can the Authors give details of the lead loss through the screens?

49. I think energy losses may be divided into

(a) the mean loss of potential between the intakes and the pump suction inlets

(b) the losses corresponding to the effect on the efficiency of the various pumps of flow disturbances at the suction inlets.

Susceptibility of pumps to disturbed suction conditions tends to be more marked the higher the specific speed.

50. Could the Authors explain why turbulent energy is assumed to be approximately proportional to the square of the (presumably streamwise component of the) velocity fluctuations? I am inclined to think the assumption is unrealistic for the following reasons.

51. For simplicity a flow regime is assumed to consist of forced vortices (i.e. vortical velocities $\lambda v$ proportional to radial distance from the axis) with vertical axis, combined with a uniform horizontal translational motion carrying the vortices past the probes.

52. The 'turbulent energy' mentioned by the Authors $(\$ 18)$ is assumed to mean the excess (shortfall) of the kinetic energy per unit mass over that corresponding to the uniform translational flow (without vortices). The probe readings are assumed to indicate in every case the streamwise component of the fluid velocity at the probe. Two cases are chosen at random

(a) where the path (in plan) traced by the vertical axis of each moving vortex is parallel to the streamwise direction and passes through the position of the probe

(b) where the path (in plan) traced by the vertical axis of each moving vortex is inclined to the streamwise direction and passes on one side or the other of the position of the probe.

53. In each case the velocity reading $r$ of the probe is considered at time $t_{1}$, when the cylindrical surface of the vortex first reaches the probe $r_{1}$; at time $t_{2}$, when the axis of the vortex is at, or at its nearest approach to, the probe $r_{2}$; and at time $t_{3}$, when the surface of the vortex leaves the probe $r_{3}$. The vortical velocity at the surface of the vortex is denoted by $v$. At a distance $\lambda r$, less than the radius, the (forced) vortical velocity is taken at $\lambda v$. The translational velocity is denoted by $\operatorname{csec} \theta$, where $\theta$ is the angle between it and the streamwise direction. (In case (a) the translational velocity is $c$.) The absolute velocity is denoted by $w$. Thus the Authors' turbulent energy is ( $w^{2}-$ $\left.c^{2} \sec ^{2} \theta\right) / 2 g$ per unit mass.

54. In case (a), at time $t_{1}$, the turbulent energy at the probe is $\left(w^{2}-c^{2}\right) / 2 g$, i.e. $\left(v^{2}+c^{2}-c^{2}\right) / 2 g$, i.e. $v^{2} / 2 g$ per unit mass; at $t_{2}$ (the central vortical velocity being zero) it is zero; at $t_{3}$ it is again $v^{2} / 2 g$. The readings of the probes $r$ are in each case $c$, because the probes are insensitive to motion perpendicular to the streamwise motion. The fluctuation of the probe readings is therefore zero and does not represent the turbulent energy except at time $t_{2}$.

55. In case $(b)$ the matter is more complicated, but Fig. 19 shows that the square of the fluctuations in the probe readings, evidenced by the differences between $r_{1}, r_{2}$ and $r_{3}$, cannot be taken as proportional to the turbulent energy given by $\left(w^{2}-c^{2} \sec ^{2} \theta\right) / 2 g$ either instantaneously or over a period.

56. Had the path of the moving vortex axis passed the probe on the other side, the velocities and so on would have been quite different. Actual conditions, usually with vortex axes inclined to the vertical, are far more complicated still. A more realistic indication of the goodness or badness of the flow might be achieved by the use, in place of propeller probes, of small-orifice impact-type probes, working on the pitot principle. 
Much research has been done on the sensitivity of these to velocities having a range of direction.

57. The concept of frequency seems to have no direct rapport with the velocity phenomena considered in the Paper. Assuming a given mean translational flow velocity, however, there may be a relationship between frequency and the size of the passing vortices.

\section{Mr A. R. B. Edgecombe, F}

Of major importance is the discharge. One must assume, however, that the terms of reference for the model did not include the assessment of discharge to be pumped. 36 cumecs for the irrigation of 46720 ha of wheat and maize assumes a duty of 1298 ha per cumec. A realistic duty would be 2030 ha per cumec, this figure being the average of four consecutive years of recorded rabi duties based on supplies utilized on the Ramganga and Gogra pumped canals in the United Provinces of India. ${ }^{20}$ Wheat is the main rabi crop. On this basis the discharge would be $46720 / 2030=23$ cumecs.

59. Did the Authors consider the use of guide walls, spaced to ensure a balanced supply to each pump, and fewer units?

\section{Dr J. D. Burton, Universidad Pontificia Bolivariana}

The deliberate creation of vorticity by columns and sills so as to control and improve the flow conditions before pump intakes is most interesting. Neglecting surface and side wall effects, one wonders if a sill upstream of the column might be equally effective? A sill may be regarded as a half column laid horizontally with the floor of the channel forming the central streamline.

61. Mention is made of spectral analysis ( $\$ 17-22)$, the results of which are not presented because of certain experimental weaknesses, and yet these spectral results are referred to again to justify partially the confidence in predicting the prototype performance $(\S 36)$ of these deliberately induced viscous mechanisms. Would the Authors comment on this please?

\section{Mr A. P. Albuquerque, Water and Sewerage Co. (CEDAE), Rio de Janeiro}

The Authors have developed an interesting solution for problems of separation in channel transitions. It is expected that this type of solution may have many other applications in engineering, e.g. entrances to sedimentation tanks.

63. I have performed similar experiments in a much smaller model with flow visualization and velocity measurements. ${ }^{31}$ It was found that the vortex breakdown or decay was a result of the many kinds of interaction between the eddies, the elongation of the eddies due to acceleration (with or without a retardation downstream) and viscous shear. The columns, which were placed at the entrance of the diffuser or lateral intake, had the function of dividing the flow and adding momentum to overcome separation in the expanding channel. It was found that the larger the number of columns the better the diffuser performance for a given degree of obstruction. The optimum number of columns must be found taking into account the degree of obstruction and the strength of the smaller columns. With more than two columns it was possible to improve the mixing process by staggering the columns to increase wake interference.

64. I have tested three types of horizontal obstruction: the sill, the ascending step and the descending step. Experiments have shown the ascending step to be the most efficient of these features, as it could be said to act as a localized source of vorticity dissipation equivalent to a length of channel. The sill had little influence, for the roller was found to play a negligible part in the process and even a ramp in front of the ascending step seemed to give good results. Comparing these observations with those of the Authors, the apparent differences may be due to different inlet turbulence levels in the models. 
65. The roller which was formed behind the sill might be capable of producing a slight bending and stretching of the vortex tubes, but I cannot agree with the tendency of the vortex lines to be inclined towards the horizontal as shown in Fig. 18. This was not observed and seems to lack a feasible theoretical explanation. In the energy spectra (Fig. 17) the Authors omitted to mention the strong constraining effect of the channel walls.

66. It seems reasonable that viscous shear and interaction between the vortices mainly in the horizontal plane are the causes for weakening or breakdown of the vortices in the wake of the columns. An ascending step placed downstream may be considered as a further help in the process, by increasing the cross-section of the vortex tubes and thus reducing their tangential velocities, as well as making the interaction between them stronger. When the ratio of height of step to depth of flow becomes large, the elongation of the cross-section of the eddies in the streamwise direction due to acceleration of the flow becomes important.

67. The Authors must be congratulated on the simplicity of their proposals, but further investigation seems necessary on the geometry of the intakes (arrangement and number of columns), their distance from the columns and the use of columns of variable cross-section. ${ }^{22,23}$

\section{Mr A. R. Thomas, F}

The design of diffusers in open channels has always been on an empirical basis and the Paper is a useful addition to published examples illustrating measures which have been successful. The Authors' theoretical background is interesting but is confined to the generation and dispersion of vortices which is only part of the relevant phenomenon, and it is not shown how the conclusions on vorticity can be applied in design. A study of other factors would be helpful. The following represents my understanding of the flow behaviour in terms of pressure differentials, transfer of momentum by turbulent mixing and Newton's second law.

69. In expanding flow kinetic energy is converted to pressure energy with a consequent rise in water level, creating a pressure gradient against the flow. This results in deceleration in the direction of flow and if a pressure gradient develops in a lateral direction it will deflect the flow. Turbulence transmits momentum laterally by interchange of fluid, whether in the form of discrete vortices or the random mixing which follows their dispersion. By Newton's second law, pressure plus momentum are in balance everywhere. Applying these principles to actual cases, consider an expansion similar to that shown in Fig. 4 and examine the stability of three alternative flow patterns.

70. In case 1 in Fig. 20 it is assumed that the flow follows the divergence on both sides. The rise of pressure in the expansion is balanced by the decrease in forward momentum. Pressure is rapidly equalized over the cross-section, whereas velocity and hence momentum are not uniformly distributed, being less near the sides than in the centre, so deceleration is relatively greater at the sides. In gradual expansions this results in an exaggeration of the non-uniformity of the velocity distribution, but where the expansion is sharp and therefore the pressure gradient steeper it may cause a reversal of flow at one or both sides and the formation of eddies. This action is countered by the lateral transfer of momentum from centre to sides by turbulent mixing. The turbulence is partly that already in the flow and partly generated by shear due to the lateral velocity gradient. A balance is achieved when the rate of momentum transferred equals the force due to the pressure gradient plus or minus boundary drag. If the expansion is gradual or the vorticity high, the expanding flow is everywhere maintained against the adverse pressure gradient and the pattern is stable. If the expansion is sharp and the pressure gradient therefore relatively steep, the flow pattern will change to that of case 2 or case 3 in Fig. 20.

71. In case 2 the eddies are symmetrical. This flow pattern occurs where the divergence is too sharp for the flow to follow on either side. The adverse pressure gradient 

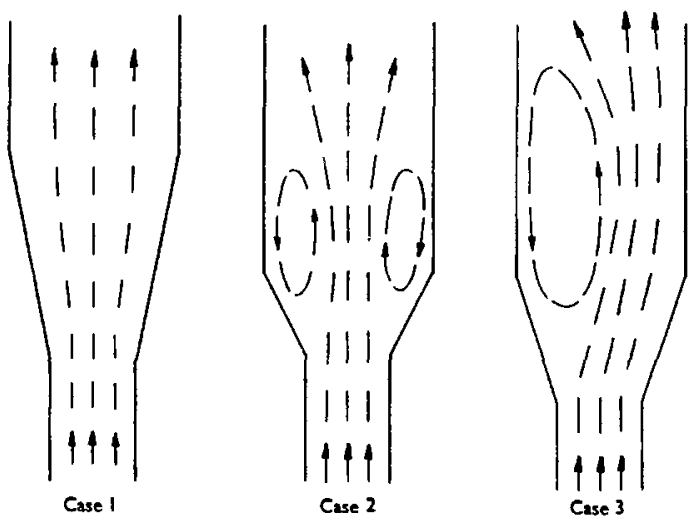

Fig. 20

promotes upstream flow at the side walls while momentum transfer from the main flow promotes downstream flow in the eddies adjacent to it. The resultant moments stimulate rotation. The eddies are stable when the upstream and downstream flows within them are equal. If an eddy is too long, the pressure gradient is unable to maintain the upstream flow and the eddy shortens and so steepens the gradient. If the eddy is too small the upstream flow is excessive and the eddy lengthens until a balance is attained.

72. Case 3 occurs when an eddy can exist on one side but not on both sides simultaneously because of the geometry. If with the pattern of case 2 the divergence were made more gradual, the eddies would become elongated, confined between the main flow and side walls and therefore subjected to a reduced pressure gradient but increased input of momentum from the main flow. Elongated eddies are less efficient because of internal shear and both would tend to be reduced in size, but if due to a small asymmetry one were to remain larger than the other, pressure would be higher on that side, deflecting the main flow to the opposite side. Its width would then be relatively greater and, given the right conditions, it could exist while the eddy on the other side is eliminated. Thus one eddy could exist more easily than two.

73. This is a simplified explanation of what has been seen to occur. It endorses at least three ways of inducing flow to follow expansions; reducing the angle of divergence, reducing the adverse pressure gradient and creating vorticity or turbulence in the expanding flow. In experiments with flow in open channel expansions, like the Authors, I found that a grid to create turbulence, a baffle or floor sill and floor blocks were all helpful in causing flow to expand. ${ }^{24} \mathrm{~A}$ grid is effective but has a practical drawback in some situations in that it collects debris. The floor blocks, like the Authors' pillars, acted in two ways; they reduced the pressure differential and generated vorticity and turbulence. The sill was effective, not just in modifying vortices generated upstream, but used alone in its action on the flow, probably due to the equalizing effect on momentum of the converging flow it caused, as has also been found with a rising floor. Deflector walls also have been found very effective in expanding flow in a short diffuser, but they may not be suitable for pumping bays because they are not adaptable to variable combinations of pump operation.

74. All this is qualitative but may be useful in producing preliminary designs for model tests which are still required in many cases. I agree that, provided a model is large enough to be well within the turbulent range, there should be no significant scale effect. Nevertheless details of the full-scale results would be of interest. 


\section{Mr Spurr}

Mr Thomas describes the flow situation in the more classically established pressuremomentun concepts, which serve very well to emphasize the difference between two basic ways of conceptual thinking. Although in principle both approaches are always valid, it is sometimes more useful to think of real fluids in terms of vorticity, as was the case in this study.

76. The central interest of the Paper lay in the understanding of the mechanics of the generation/transport/disorganization of the rotational secondary flows, deliberately developed to overcome their normally undesirable counterparts, as well as to enable prediction of the functioning of the 36 prototypes with a reasonable degree of certitude.

77. The classical difficulty arising from the momentum approach ( $\$ 69$ and 70 ) is that momentum is assumed to be a transferable property and necessarily involves the accompanying assumption that the fluctuating variations in pressure, which always exist in turbulent shear flow, are ineffective so far as the mean transport of momentum is concerned. This is, for the case in question, simply not so. For direct comparison, a brief description of the essential functioning of this class of flow, in terms of vorticity, is given ${ }^{25,26}$ as follows.

78. The relative velocities within a shear flow field are considered to be induced by viscous and turbulent shear stresses rather than by pressure gradients, so that these flows are thought of as rotational rather than potential, wherein micro-turbulent motions are generated and are self-sustaining. Shearing motions are produced in line with the shear stresses and generally conform to the shape of the boundaries, whereas the irregular secondary motions (turbulence) do not depend on the shape of the boundaries but rather, like all secondary flows, depend on the generating mechanisms which produce motions in directions other than that of the applied shear.

79. Even though the energizing of the turbulence may be expressed in terms of the shear stresses, turbulent pressure gradients do arise from within the flow itself and result in interaction between the fluid elements. These interactions may perhaps best be described by considering the shear flow field as a matrix superimposed throughout with numerous eddies of various sizes and orientations, which either combine and grow or break down into smaller ones, depending on their organization ( $\$ 23$ ).

80. In the momentum approach, diffusion may be thought of as analogous to the conduction of heat by molecular collisions, where the transfer is through the conversion of kinetic to potential energy. In the vorticity approach, diffusion is thought of in terms of the deformation of the vortex structure, so that the transfer is thought of in terms of straining process (strain energy).

81. The Paper was presented in these conceptual terms, which were not only helpful to the practical understanding of the generation and organization of the shear flows (\$\$24-32), but also vital to the attempts to understand and describe the mechanisms responsible for the rapid disorganization of their complex structures, in order to enable sensible prediction of the functioning of the 36 prototypes (\$33-36).

82. It is my opinion that vortex bursting was the dominant operative mechanism in bringing about the rapid lateral diffusion of energy responsible for destruction of the wake structures. This seems to be concurrent with the conceptual Standford model of turbulent boundary layers referred to by Jackson ${ }^{27}$ in his survey of visualization studies.

83. Both Dr Burton and Mr Lupton query the relevance of the spectral approach. As implied in the Paper $(\$ \S 17,18$ and 30 ), the fundamental purpose of the spectral analyses was to describe the macro-energy state of the station entry flow, thereby enabling examination of two very important features of the model tests (in that testing techniques were valid).

84. Comparison of the forms of the spectral energy with frequency distribution of the idealized fully diffused flow to that of the turbulent subsurface flows recorded at the station entrances gives, in principle, a good indication of the actual degree of turbulent diffusion/mixing achieved upstream of the sampling point. 


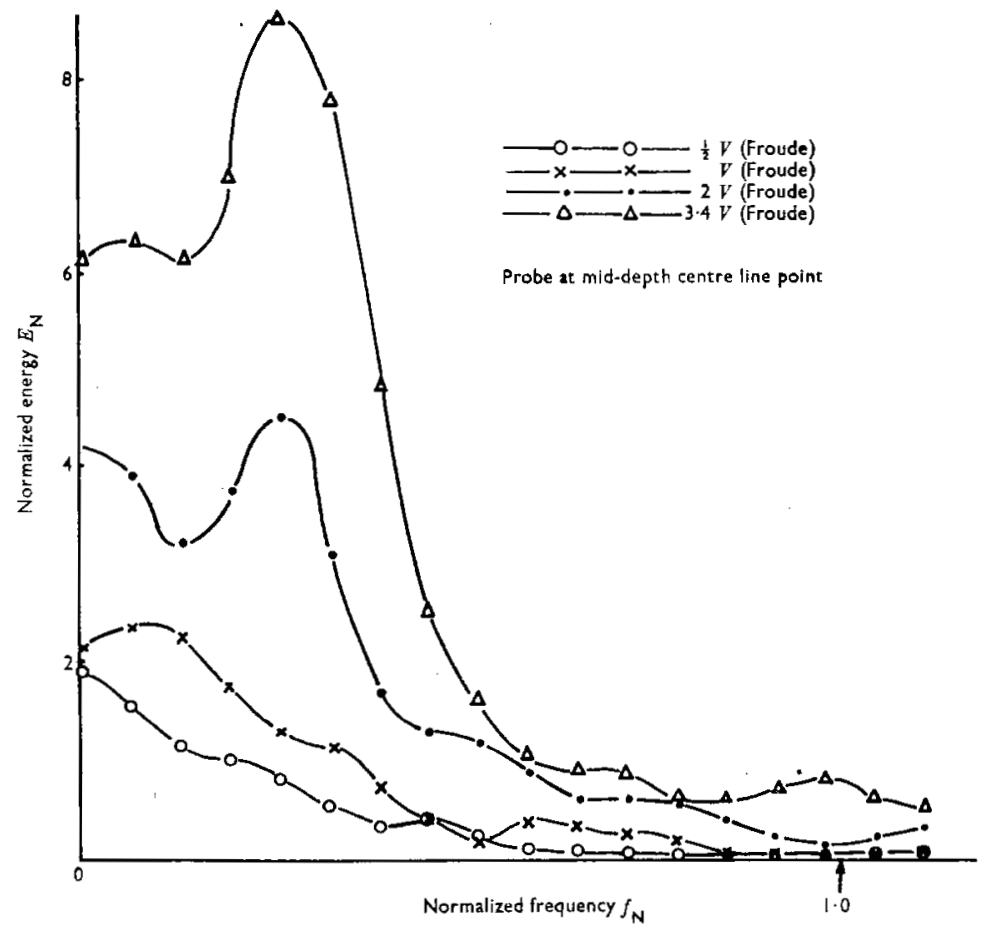

Fig. 21. Station SRP1, bay 1 (see Fig. 4)

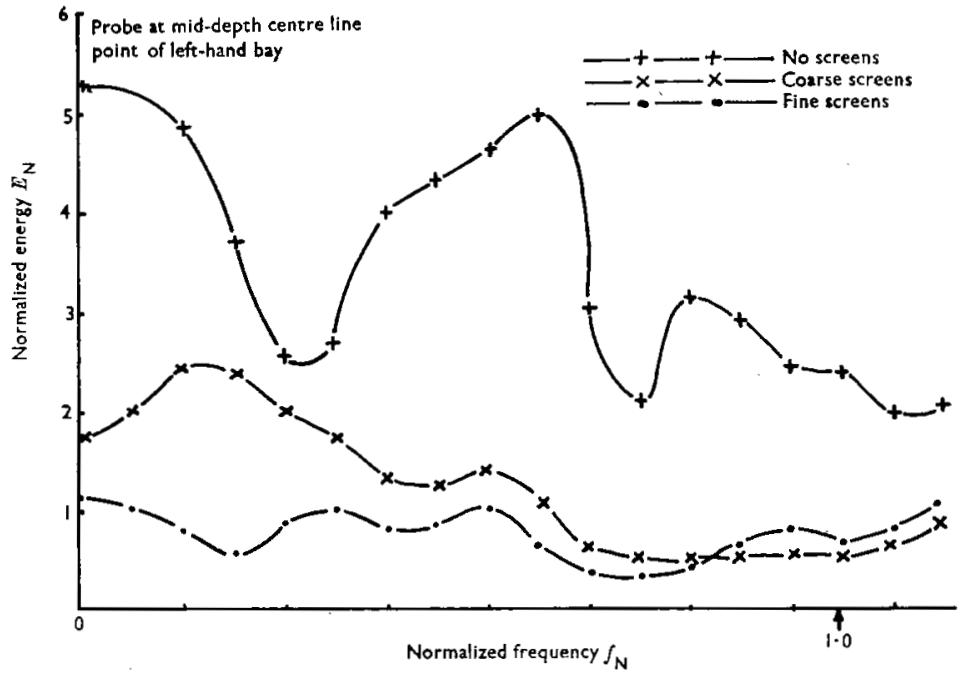

Fig. 22. Station PPS at $1 \times$ Froude velocity (see Fig. 16) 
85. It was considered reasonable to suppose that the steady state design flow within the irrigation distribution canals would be the best practical condition achievable. By normalizing and expressing all spectral forms as multiples of the spectral peak energy value and frequency of occurrence of any typical distribution canal flow, it was postulated that a reasonable qualitative measure of the acceptability of the flow condition at the station entrances may be seen by comparing the differences in the recorded spectral energy ordinates with its corresponding fully diffused, idealized form (Figs 17 and 21), taking into account the actual smoothing effects brought about by the screens (Fig. 22).

86. Pertinent to basic modelling philosophy and in order to establish that the viscous dependent, kinetic secondary flows (so relevant to the wake structure) had been significantly simulated, the model velocities were simply increased above the Froude criterion velocity so as to simulate better the rotational elements within the overall flow structure. By so doing, it was sought to determine if a significant change in the basic flow organization would occur as the kinetic momentum/viscous force ratio increased. This would be indicated by corresponding changes in the surface flow patterns and/or more certainly by changes in the forms of the subsurface spectral energy distribution.

87. Since the flow regime in the mixing region between the sill and the station may be assumed to be a turbulent boundary layer flow, it can be seen that interest lay in the comparative states of the low frequency, macro-turbulent energy forms rather than in any quantitative analysis of the energy spectrum as a whole.

88. The spectral results presented in Fig. 21 serve to illustrate this approach. Although no significant changes in the water surface patterns with increase in velocity were observed, a basic change in the form of the spectral energy distribution in the low frequency (macro-energy) ranges exists below and above the Froude criterion. Further increases in velocity do not appear to alter its basic form thereafter. The lengths of the velocity with time traces recorded were chosen to fulfil approximately the statistical criterion stipulated by Iwasa. ${ }^{2}$

89. I can only partially agree with Mr Lupton's scepticism concerning the inherent unsuitability of the use of mini-flow propeller probes for these particular spectral purposes. I do not agree with his mention of forced vortices but rather suppose he refers to free vortices, since wakes are a class of free turbulent shear flows. It is also not strictly correct to assume that the propeller probes measured only the streamwise components, since those used were of the five-blade helical type, with a characteristic directional sensitivity to yaw angles of approximately $\pm 15^{\circ}$. However, it is true that they are relatively insensitive to large lateral flow movements and a directionally more sensitive probe would have been better employed had one been available. The probes were located immediately upstream of the station screens at the centre line mid-depth position of typical individual pumping bays, and were aligned face on to the direction of the flow so as to measure essentially the mainstream flow and its fluctuating components (for example, model SRP1, bays 1 and 4, Figs 2, 4 and 21). Details of actual readings taken are regrettably not readily available.

90. Although large-scale, organized wake structures are known to be anisotropic, the flow arriving at the station entrances was seen to be largely disorganized. In view of its chaotic nature and nearly random state as well as for reasons of practical simplicity, the streamwise direction was simplistically assumed to be characteristic and the turbulent energy to be approximately proportional to the square of the streamwise velocity components. Nevertheless, the interest was essentially qualitative, exclusive of the micro-turbulent energy, and even though a larger degree of inaccuracy than is normally associated with spectral analyses could be tolerated, the spectral results were not presented in the Paper.

91. Two attempts to assess the effect of vortex modifying devices on pump efficiencies have been made by Labetoulle and Berge. ${ }^{28,28}$ Perhaps such information would be obtainable from a large pump manufacturer. Head losses through the different screens are not available, although comparison of the order of magnitude of their 
smoothing effect on the entry flow is shown spectrally in Fig. 22. The probe was placed at the centre line mid-depth ten probe diameters downstream of the screens of a typical PPS pump bay.

92. Mr Edgecombe's contribution is interesting. The definition of the water requirements and basic project layouts were stipulated by the Romanian Agricultural Authorities and were outside the terms of reference of this study.

93. Combinations of flow-dividing walls projecting from the station entrances into the canal forebay area were tried in model SRP1, but were found to be unsuitable because they gave rise to surging and poor flow distribution across the entrances at partial station operation.

94. Dr Burton's suggestion of reversing the column-sill order was tried briefly during the tests. The sill alone was found to be locally effective in preventing separation at the canal side walls since its upstream face caused the flow to be deflected laterally, increasing the lateral convection of energy. The addition of two columns downstream of the sill not only prevented side wall separation, but also in so doing created column wakes which flowed undesirably straight into the station entrances. The sill was finally positioned downstream of the columns when it was seen that it not only helped overcome the side wall separation but was also instrumental in bringing about the disorganization of the column wakes. This combination has the advantage that the distance between the columns and downstream sill may be adjusted accordingly for different lengths of diffuser.

95. Mr Albuquerque's model findings, indicating that the roller appears to play no direct part in the wake destruction, are very interesting. From a comparison of the ratio of the scales and Reynolds numbers involved

$\begin{array}{lccc}\text { Linear scale } & 1: 1 & 1: 12 & 1: 45 \text { approx. } \\ \text { Reynolds number } & 0.9 \times 10^{6} & 0.2 \times 10^{5} & 0.4 \times 10^{3}\end{array}$

it may be seen that the representation of viscous forces in the 1:45 model was approximately two orders of magnitude larger than those present in the prototype and yet both models and prototype functioned satisfactorily. One possible explanation ( $\$ 35)$ is that the balance between the interacting kinetic momentum forces remained approximately the same whatever the scaling error.

96. I do not share Mr Albuquerque's opinion that the difference in the simulation of the background turbulence levels was significant, since the discrepancies are only likely to have affected the micro-turbulent and dissipation processes which were thought to be of little consequence to the overall diffusion disorganization of the wake ( $\$ 34$ and 35 ).

97. Perhaps more may be deduced from Jackson ${ }^{27}$ and Grabowsky and Berger ${ }^{30}$ who showed that the vortex breakdown phenomenon is not just peculiar to supercritical rotating flows having a swirl value very close to the critical swirl value, but may and commonly do exist in many subcritical flow regimes. Visualization studies ${ }^{27}$ indicate that vortex bursting is the dominant mechanism in the fluid transfer processes within turbulent boundary layers. Numerically it has been shown that for axisymmetric vortices ${ }^{30}$ the disturbance of the sensitive, non-linear coupling of the axial to swirl velocities of the vortical motion can lead to the rapid onset of breakdown and accompanying high rates of diffusion.

98. It would therefore seem that each of the three configurations tried in the 1:45 model combines one or more of the basic and necessary conditions to bring about instability in the axial-swirl coupling. As to what actually caused the instability in each of the three specific configurations-a sudden reduction in the swirl velocity through neighbour interaction, a rapid change in vortex cross-section or reduction of axial core velocity due to adverse pressure gradients-is too complex to say.

99. The inclination of the vortex lines in Fig. 18 was approximated from potential flow considerations and is no more than a conceptual illustration. No attempt was made to include or superimpose the effects of the complex interactions of the lateral and streamwise vortex elements. 


\section{References}

20. UNITED PROVINCES. Irrigation administration report for the years ending March 31st, 1939-42. Allahabad.

21. Albuquerque A. P. The design of short open-channel diffusers. MSc thesis, Imperial College, London, 1975.

22. Gerrard J. H. The three-dimensional structure of the wake of a circular cylinder. J. Fluid Mech., 1966, 25, Part I, 143-164.

23. TANEDA S. Experimental investigation of vortex streets. J. Phys. Soc. Japan, 1975, 20, No. 9, Sept., 1714-1721.

24. Thomas A. R. Flow in expansions in open channels. Punjab engineering congress, 1940, 28, Paper 236.

25. MCQUIVEY S. Principles and measuring techniques of turbulence characteristics in open channel flows. Prof. Pap. U.S. Geol. Surv., 1973, No. 802.

26. HiNZE J. O. Turbulence, 2nd ed. McGraw-Hill, 1975; chapters 5 and 6.

27. JACKSON R. O. Sedimentological and fluid dynamic implications of turbulent bursting phenomenon in geophysical flows. J. Fluid Mech., 1976, 77, Nov., $531-560$.

28. Berge J. P. Enquête sur la formation de vortex et autres anomalies d'écoulement dans une enceinte avec ou sans surface libre. Houille Blanche, 1966, No. 1, 13-40.

29. Labetoulle J. and Berge J. P. Suppression des vortex dans les chambres d'aspiration des pompes de circulation de la Centrale thermique de Creil St.-Leu. Houille Blanche, 1958, B spécial, 732-743.

30. Grabowski W. J. and Berger S. A. Solution of the Navier Stokes equations for vortex breakdown. J. Fluid Mech., 1976, Part 3, 525-544. 